\title{
Diversidad estacional de la clase Bacillariophyceae en el río Utcubamba, Perú
}

\section{Seasonal diversity of the Bacillariophyceae class in the river Utcubamba, Peru}

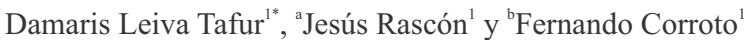

\section{RESUMEN}

Las diatomeas en áreas tropicales son una importante herramienta de biomonitoreo de la calidad ecológica del agua gracias a sus características fisiológicas y morfológicas. En la presente investigación se realizó un inventario de las diatomeas existentes en la cuenca del río Utcubamba, en el Departamento de Amazonas, durante dos épocas (seca y húmeda) caracterizadas por poseer grandes diferencias estacionales en cuanto al régimen de precipitaciones. Se registraron 112 especies distribuidas en 21 familias y 25 géneros, siendo el género Gomphonema el más diverso con 18 especies identificadas, además de ser el más abúndate, con un $26 \%$ del total de individuos. De la misma forma, la especie Gomphonema rhombicum fue la más abundante con un 17\% respeto al total de registros. Las dos épocas muestreadas mostraron resultados distintos tanto en diversidad como en abundancia de diatomeas, presentando la época seca valores superiores en ambas variables influidos por factores de perturbación de origen climático.

Palabras clave: Diatomeas, inventario, abundancia, áreas tropicales.

\begin{abstract}
Diatoms in tropical areas are an important biomonitoring tool for the ecological quality of water due to its physiological and morphological characteristics. In the present investigation an inventory of the diatoms existing in the Utcubamba river basin in the Department of Amazonas was carried out during two periods (dry and wet) characterized by great seasonal differences in precipitation regime. 112 species were recorded in 21 families and 25 genera, being the genus Gomphonema the most diverse with 18 species identified, besides being the most abundant, with $26 \%$ of the total of individuals. In the same way, the species Gomphonema rhombicum was the most abundant with a $17 \%$ respect to the total of records. The two sampled seasons showed different results in both diversity and abundance of diatoms, showing the dry season higher values in both variables influenced by climatic disturbance factors.
\end{abstract}

Keywords: Diatoms, inventory, abundance, tropical areas.

\footnotetext{
${ }^{1}$ Universidad Nacional Toribio Rodríguez de Mendoza de Amazonas (UNTRM-A), Instituto de Investigación para el Desarrollo Sustentable de Ceja de Selva, Calle Higos Urco N³42-350-356, Calle Universitaria No 304, Chachapoyas, Perú

a E-mail:jesus.rason@untrm.edu.pe ${ }^{b} E-m a i l:$ fernando.corroto@untrm.edu.pe

"Autor de correspondencia: E-mail: damaris.leiva@iuntrm.pe
} 


\section{INTRODUCCIÓN}

Las diatomeas, algas fitobentónicas pertenecientes a la clase Bacillariophyceae, suelen constituir el 80$90 \%$ de la comunidad del perifiton en los ríos. Se caracterizan por tener una gran diversidad, pues presentan distintas tolerancias frente a la contaminación (Ciutti, 2005), además de una distribución amplia a nivel mundial. En este sentido, son las algas más utilizadas como biomonitores, debido a su adaptabilidad, elevada tasa de reproducción (CHE, 2005) y por su función en los procesos ecológicos. Son la base de la trama trófica debido a su condición de productores primarios (Díaz y Maidana, 2005), de tal forma que a las variaciones de los nutrientes así como de factores como luz, temperatura, $\mathrm{pH}$, velocidad de la corriente, fósforo en el agua, y materia orgánica, responden mediante cambios en su composición, disminución de su biodiversidad o incremento de la biomasa (CHD, 2010). Las diatomeas como bioindicadores tiene una ventaja adicional y esto se debe a que su esqueleto de sílice conocido como frústulo es de elevada resistencia; además, las características morfológicas que presenta este frústulo son la base de la identificación de especies, por lo que la manipulación y conservación de las muestras resulta relativamente sencilla (Blanco et al., 2004)

Teniendo en cuenta estas características, existen investigaciones de índole internacional que buscan definir la tolerancia de algunas especies, recurriendo inicialmente al desarrollo y establecimiento de inventarios biológicos (Céspedes-Vargas et al., 2016). Estos resultan comunes cuando se elaboran índices, pues se necesita considerar la información autoecológica de los taxones y su dominancia. Es el caso del continente europeo, que ha desarrollado múltiples índices biológicos usando diatomeas, como el índice de polusensibilidad específica (IPS) o el índice biológico de diatomeas (IBD), entre los más comúnmente usados. Este modelo de investigación con indicadores biológicos representativos en sistemas acuáticos se ha trasladado al continente centro y sudamericano con el desarrollo de estudios de calidad de aguas usando diatomeas como organismo central (Ortiz-Castro, 2015; González, 2016; Vouilloud et al., 2016). Sin embargo, en el ámbito nacional existen pocos estudios estrictamente relacionados, como el desarrollado por Yupanqui y Quispe (2014) en el lago Titicaca, quienes registraron del total de flora planctónica identificada, un 23,38\% de organismo de la familia Bacillariophyceae; de igual manera, Calizaya-Anco et al. (2013) realizaron una identificación de 77 taxas en el río Locumba (Departamento de Tacna), cuyos ejemplares más diversos procedieron de los géneros Nitzschia sp. y Naviculasp.

Frente a esta situación el presente estudio ha desarrollado un inventario florístico de diatomeas de la cuenca del río Utcubamba, con el objetivo de llenar el vacío de conocimiento existente en la región al respecto, de tal forma que sirva el mismo como base para el desarrollo de investigaciones posteriores o la adaptación de un índice propio en el área de estudio usando este tipo de organismos.

\section{MATERIALY MÉTODOS}

\section{Área de estudio}

El estudio se realizó en la cuenca del río Utcubamba, entre los 381 y 2834 m.s.n.m. El río Utcubamba recorre en dirección S-N el Departamento de Amazonas, atravesando cinco de las siete provincias que componen el mismo: Bagua, Utcubamba, Bongará, Luya y Chachapoyas (Figura 1).

El área total de la cuenca es de $644317 \mathrm{Ha}$, y a partir de este cálculo se establecieron 43 estaciones de muestreo, ubicadas a lo largo de la cuenca, desde las cercanías de su nacimiento hasta su desembocadura en el margen derecho del río Marañón. Estas estaciones fueron georreferenciadas según el sistema de coordenadas Universal Transversal de Mercator (UTM) (Datum WGS 84, Zona 17 y 18 Sur). De las 43 estaciones, 25 se ubicaron en el cauce principal y las 18 restantes en los principales afluentes. El período de muestreo se dividió en dos épocas, determinadas a partir del conocimiento del calendario de lluvias, definiéndose la época seca para el mes de agosto del 2014 y la época húmeda para el mes de marzo del 2015. 


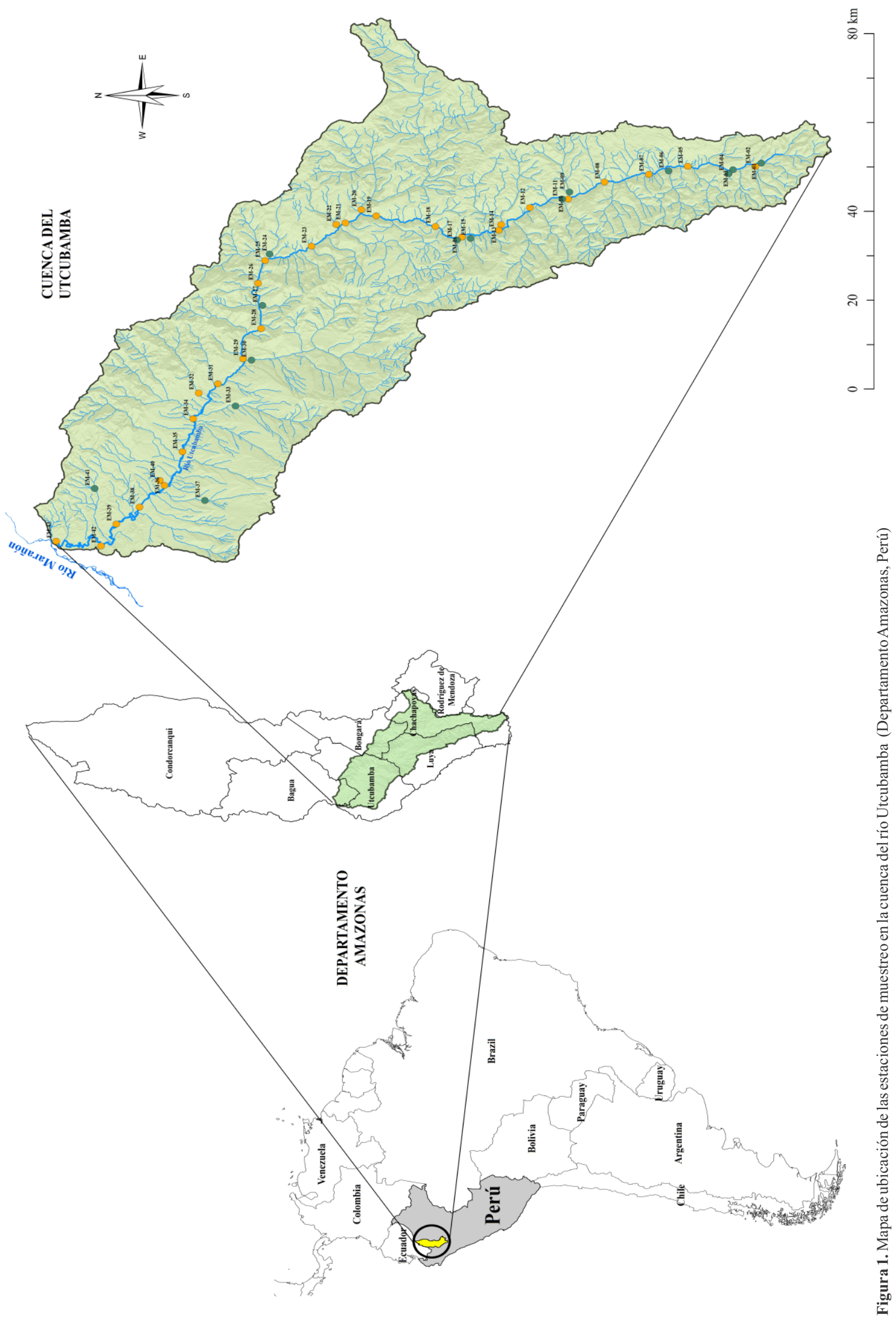




\section{Muestreo de diatomeas}

En cada estación de muestreo se colectaron cinco piedras sumergidas de entre 10 y $20 \mathrm{~cm}$, ubicadas en zonas bien iluminadas y con corriente constante. La superficie de estas piedras fue cepillada en área aproximada de $20 \mathrm{~cm}^{2}$, completando un área total de $100 \mathrm{~cm}^{2}$. El cepillo conteniendo la muestra rica en diatomeas epilíticas se introdujo en un frasco con tapa hermética conteniendo $50 \mathrm{ml}$ de agua de río. Al frasco conteniendo la muestra de diatomeas se le adicionaron seis gotas de glicerina para evitar el rompimiento de los frústulos y cinco gotas de Solución Tamponada de Formaldehido (HCHO) al 4\% para la conservación de la muestra en el tiempo.

Los frascos se etiquetaron y transportaron en frío al Laboratorio de Investigación en Suelos y Aguas de la Universidad Nacional Toribio Rodríguez de Mendoza de Amazonas (UNTRM), dejándolos en reposo por un periodo de 24 o 48 horas. Transcurrido este tiempo, se eliminó el sobrenadante y del contenido restante se extrajeron $10 \mathrm{ml}$ de muestra, que fueron sometidos a un proceso de oxidación con peróxido de hidrógeno $\left(\mathrm{H}_{2} \mathrm{O}_{2}\right)$ al $30 \%$, a razón de $20 \mathrm{ml}$ a $90^{\circ} \mathrm{C}$ durante tres horas para limpiar los frústulos y facilitar la posterior identificación.

Culminado este proceso, se dejó enfriar y se adicionaron tres gotas de ácido clorhídrico $(\mathrm{HCl}-3 \mathrm{M})$ para eliminar carbonatos presentes en la muestra. Posteriormente las muestras fueron colocadas en tubos de centrífuga, aforados a $15 \mathrm{ml}$ con agua destilada para ser centrifugadas a 30 RPM durante tres minutos. La muestra ya procesada se depositó en frascos pequeños agregando a cada uno de ellos tres gotas de Solución Tamponada de Formaldehido (HCHO) al 4\%, y se conservaron en las incubadoras a una temperatura de $4^{\circ} \mathrm{C}$.

El montaje definitivo consistió en colocar $1 \mathrm{ml}$ de muestra procesada en una cámara de conteo Sedgwick-Rafter, dejándola reposar cinco minutos para permitir que las células se depositen en el fondo de la cámara, $\mathrm{y}$ a continuación se examinaron a una magnificación inicial de 20X y una final de 40X en un microscopio óptico. Antes de iniciar el recuento, se observó detenidamente toda la extensión de la cámara para identificar las principales especies presentes en la muestra, comprobando que los organismos se dispersen al azar a través del área de conteo y no se encuentren restringidos en una región en particular.

Por último, utilizando manuales de e inventarios de diatomeas relacionados (CHD, 2010; Soler et al., 2012) se llevó a cabo la identificación de estos organismos hasta el nivel de especie.

\section{RESULTADOS}

\section{Riqueza total de especies}

Se colectaron un total de 115783 individuos correspondientes a 21 familias, 25 géneros y 112 especies de diatomeas. Los géneros más diversos fueron Gomphonema con 18 especies, Nitzschia con 15 especies, Fragilaria con 13 especies, y Navicula con 12 especies (Figura 2).

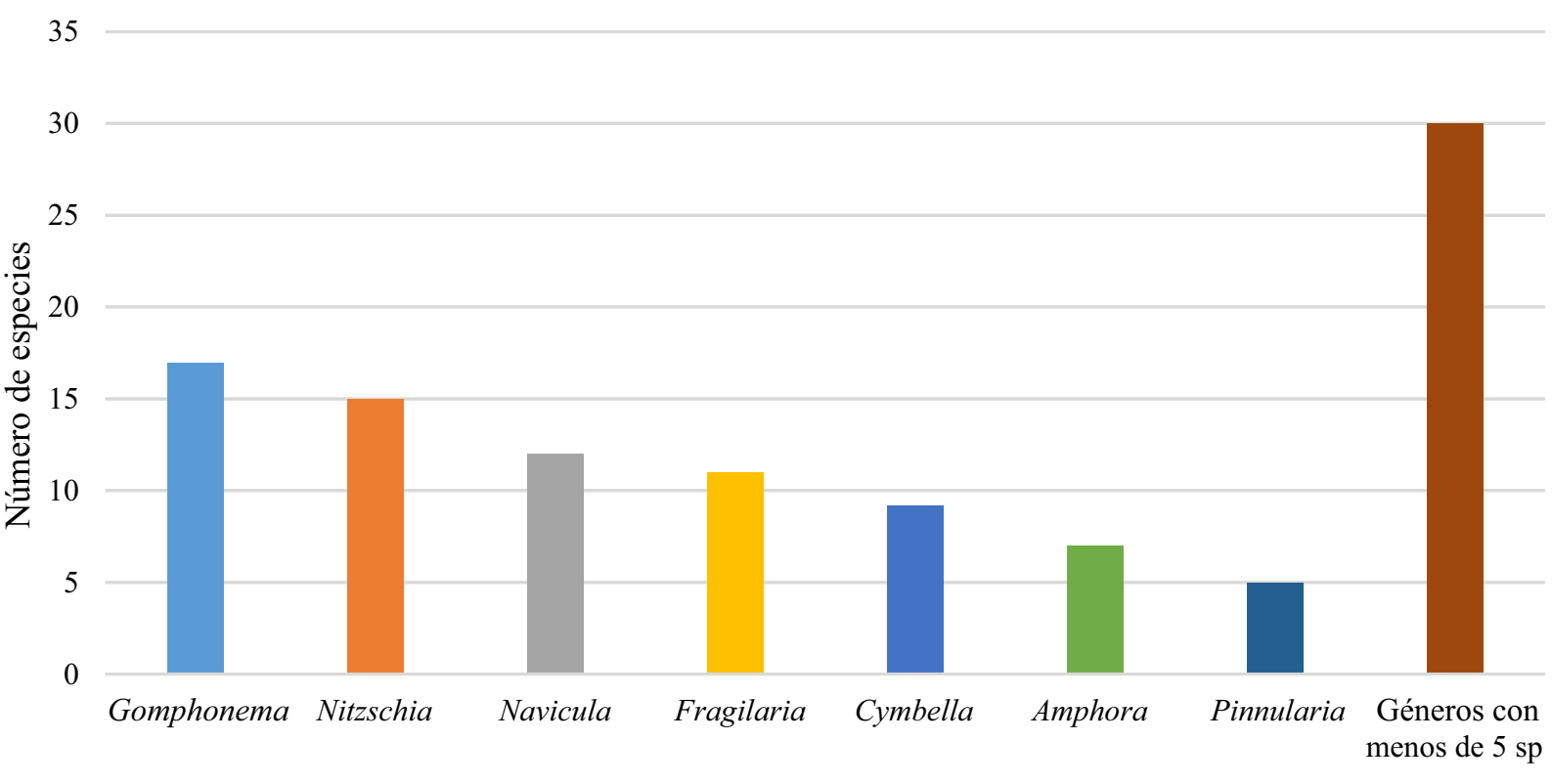

Géneros

Figura 2. Diversidad de géneros colectados en la cuenca del río Utcubamba. 
Respecto a la abundancia, las familias con mayor número de individuos fueron Gomphonemataceae, con un 26\% y Achnanthidiaceae con un 19\%, seguidas de Bacillariaceae y Naviculacea con un $16 \%$ y un $12 \%$, respectivamente (Figura 3).

En relación a los géneros, la abundancia fue similar para los que pertenecen a las familias más abundantes; así, Gomphonema, Achnanthes, Nitzschia y Navicula fueron los géneros con mayor número de individuos inventariados con $26 \%, 19 \%, 16 \%$ y $12 \%$, respectivamente (Figura 4).

En ese aspecto, las especies con mayor número de individuos inventariados fueron Gomphonema rhombicum y Achnanthes minutissia con el 17\% del total de individuos cada una, seguidas de Gomphonema pumilum var. rigidum y Nitzschia palea, ambas con un 7\%

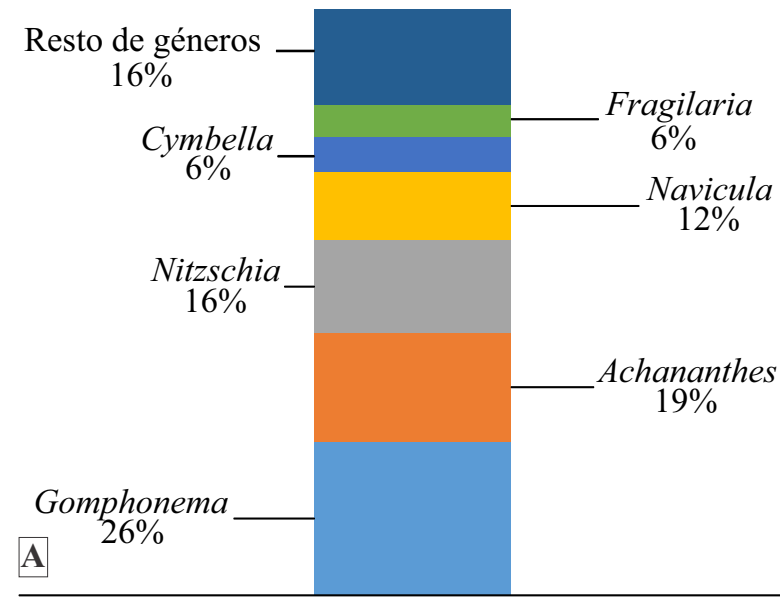

Géneros

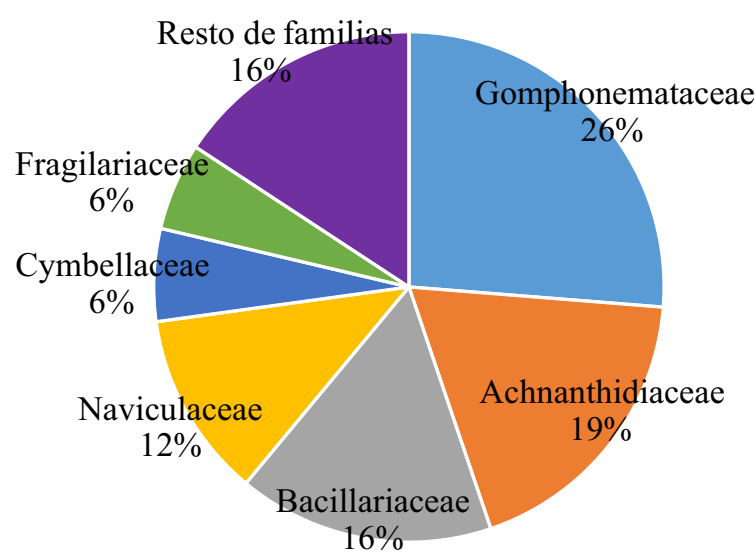

Figura 3. Abundancia de individuos en función de las familias identificadas en la cuenca del río Utcubamba.

del total, y Cocconeis euglypta y Nitzschia media, ambas con un 5\% (Figura 5).

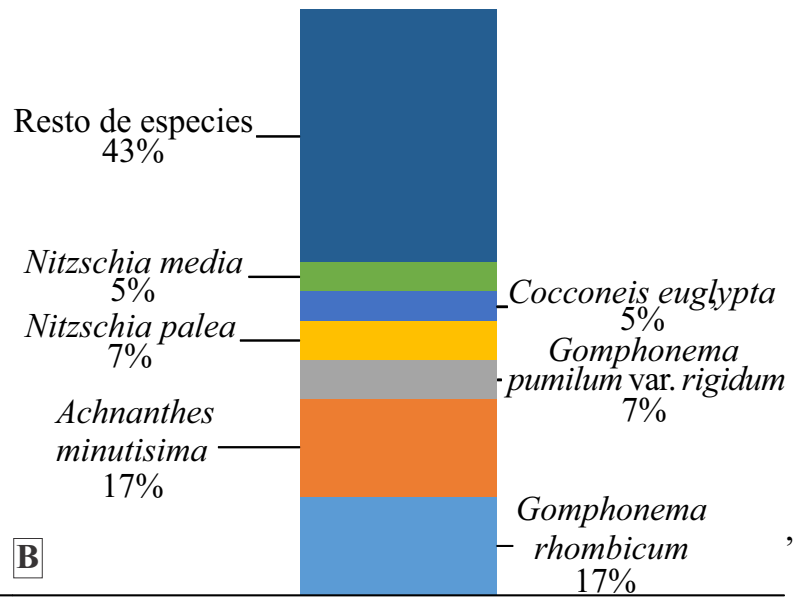

Especies

Figura 4. Abundancia de individuos en la cuenca del río Utcubamba: A. En función de los géneros identificados; B. En función de las especies identificadas.

\section{Comparativa de la riqueza de especies identificadas en época seca (Agosto, 2014) y época húmeda (Mar- zo, 2015)}

Durante la época seca se capturaron un total 74504 individuos correspondientes a 21 familias, 25 géneros y 106 especies de diatomeas. Los géneros más diversos fueron Gomphonema con 17 especies, seguido de Nitzschia con 15 especies, y Navicula y Fragilaria, ambas con 12 especies (Figura 5). Por otra parte, durante la época húmeda se capturaron un total 41279 individuos correspondientes a 20 familias, 22 géneros y 71 especies de diatomeas. Los géneros más diversos fueron
Gomphonema con 12 especies, Fragilaria y Navicula con nueve y ocho especies, respectivamente (Figura 5).

Las familias más abundantes en individuos identificadas durante la época seca fueron Gomphonemataceae con un 34\% del total, seguida de Achnanthidiaceae y Naviculaceae con un 12\% ambas, y Bacillariaceae con un $11 \%$ (Figura 6A).

Por el contrario, las familias más abundantes en individuos colectados en la época húmeda fueron Achnanthidiaceae, con un 30\% del total, seguida de Bacillariaceae, con un $26 \%$, y Gomphonemataceae y Naviculaceae, ambas con un 12\% (Figura 6B). 

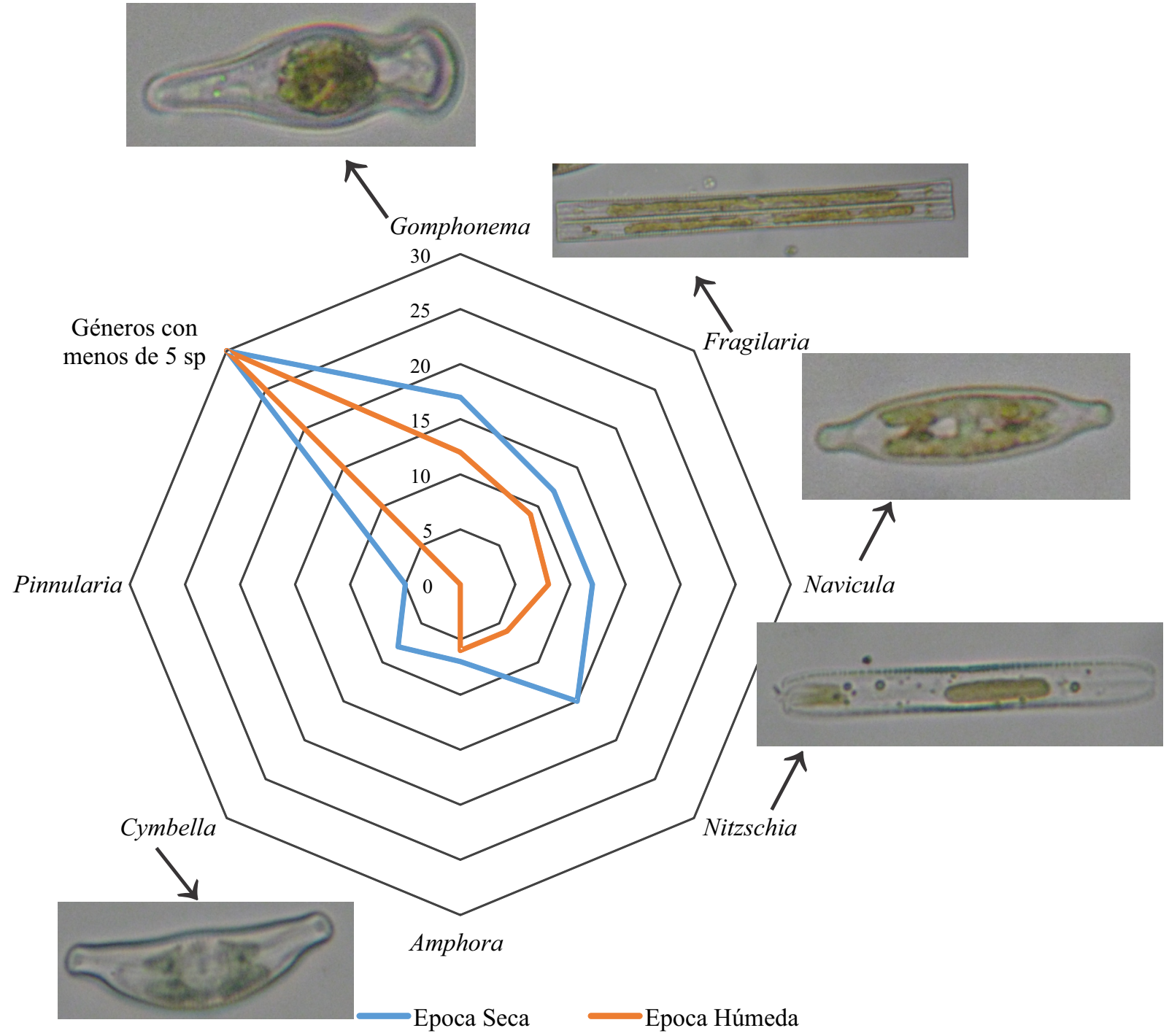

Figura 5. Diversidad de géneros colectados en la época seca y húmeda.

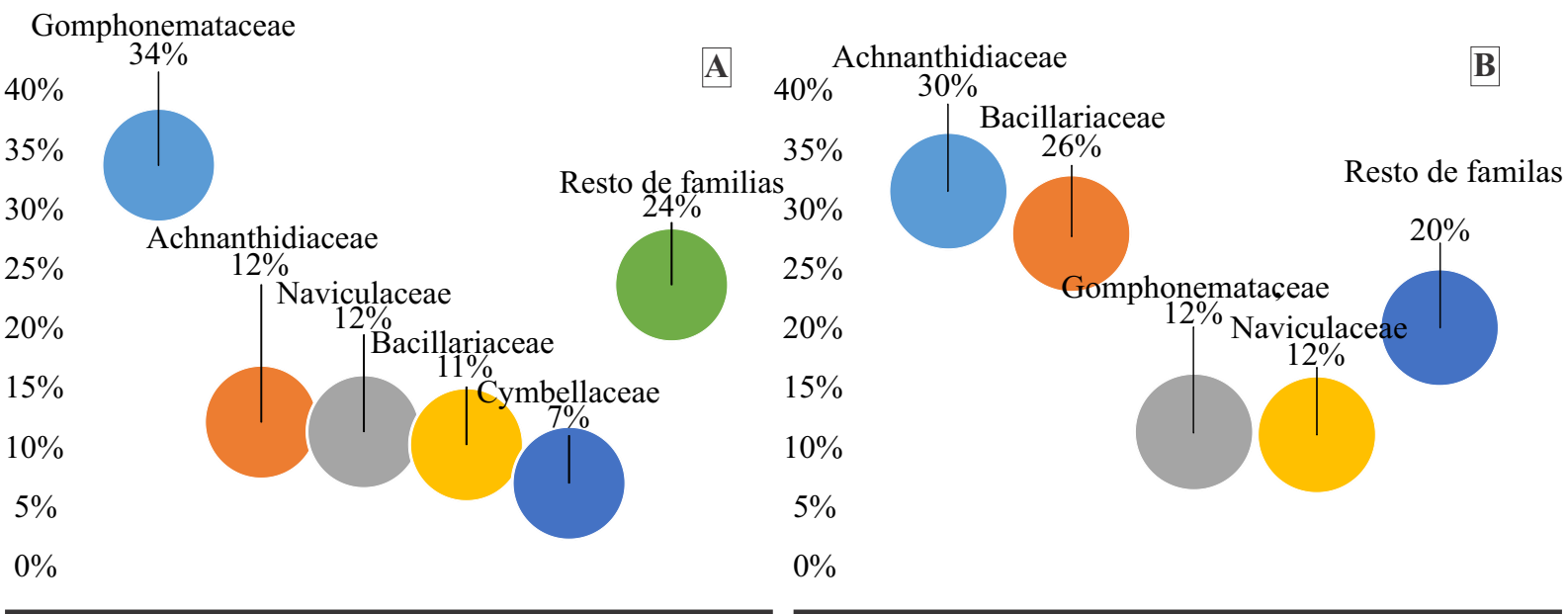

Figura 6. Abundancia de individuos en función de las familias colectadas: A. Época seca; B. Época húmeda. 
En referencia a los géneros identificados en la época seca, la abundancia fue similar para los géneros pertenecientes a las familias más abundantes, de tal manera que Gomphonema, Achnanthes, Navicula y Nitzschia resultaron como los géneros con mayor número de individuos inventariados en la época seca, con 34\%, $12 \%, 12 \%$ y $10 \%$, respectivamente. Mientras que en la época húmeda los géneros más abundantes resultaron Acharanthes y Nitzschia (Figura 7Ay 7B)

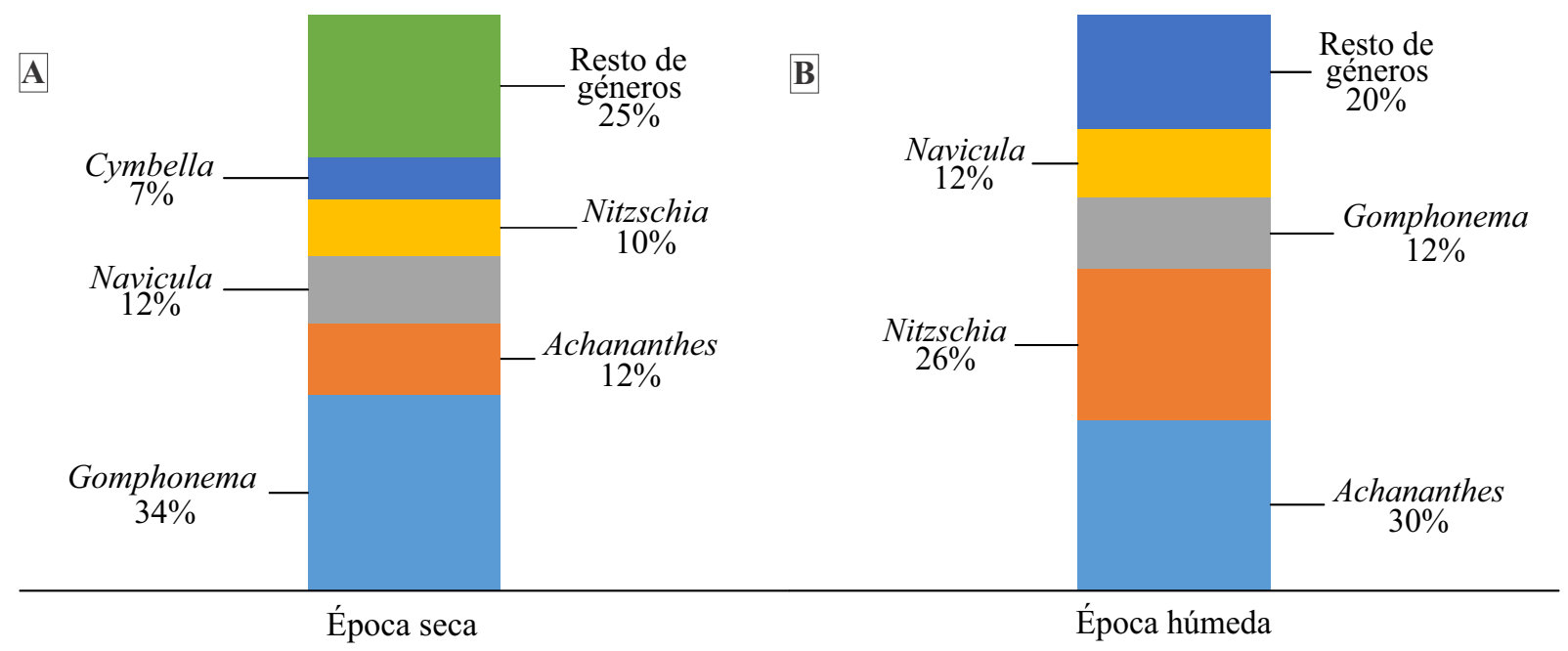

Figura 7. Abundancia de individuos en función de los géneros colectadas: A. Época seca; B. Época húmeda.

Finalmente, las especies más abundantes en número de individuos en la época seca fueron Gomphonema rhombicum con un $22 \%$ del total, seguida de Achnanthes minutissia y Gomphonema pumilum var. rigidum con un $11 \%$ y un $9 \%$, respectivamente (Figura 8). Asimismo, las especies más abundantes en número de individuos colectados en la época húmeda fueron Achnanthes minutissia, con un $27 \%$ del total, seguida de Nitzschia palea con un 13\% y Nitzchia media y Gomphonema rhombicum, ambas con un $8 \%$ del total (Figura 8).

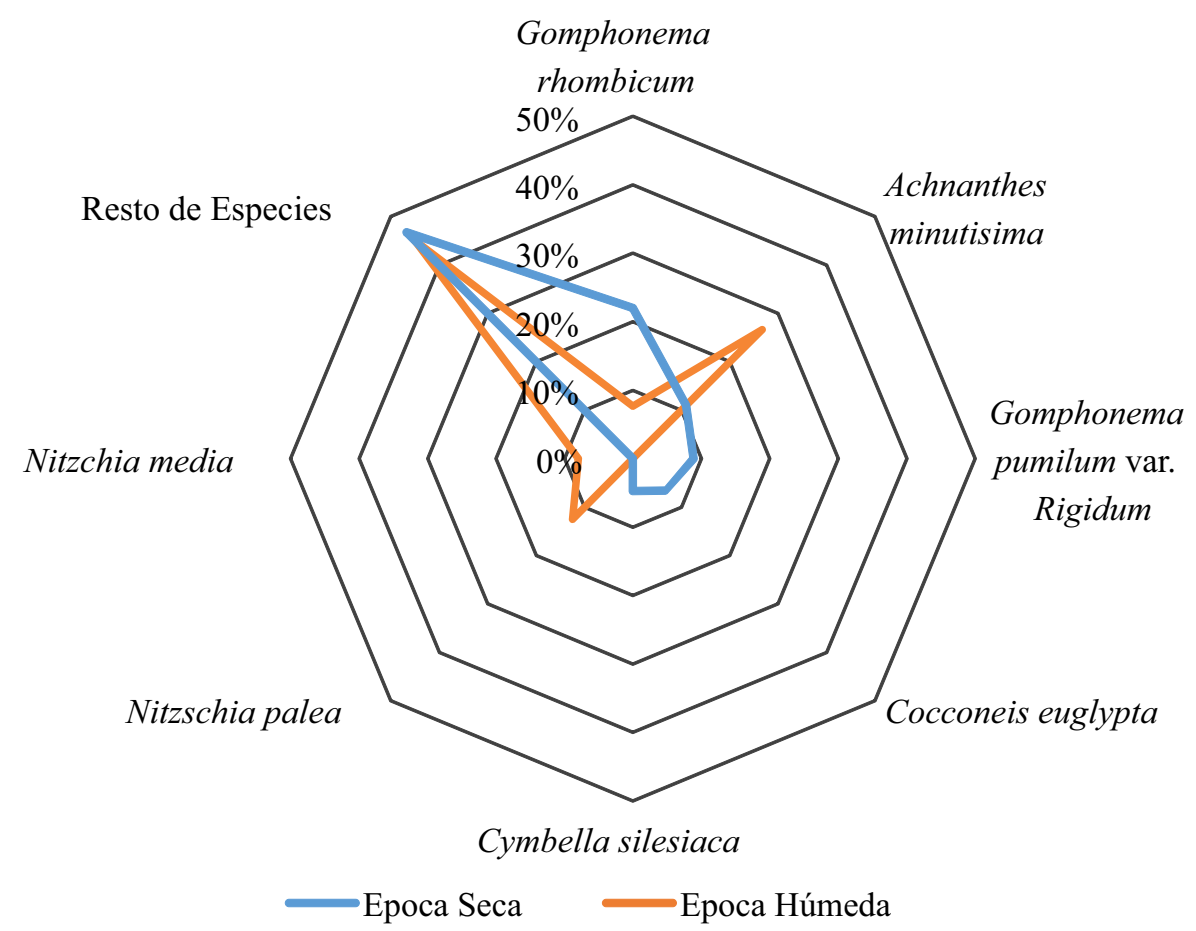

Figura 8. Abundancia de individuos en función de las especies identificados en la época seca y húmeda). 


\section{IV.DISCUSIÓN}

La diversidad de diatomeas identificadas en la cuenca del río Utcubamba se tradujo en 25 géneros colectados durante ambas épocas de muestreo (seca y húmeda). De esta manera, se encontraron diferencias entre los meses de muestreo, siendo la época húmeda la menos diversa, con 22 géneros, mientras que en la época seca se identificaron 25 géneros. Esto se debe a una de las características con más peso en este tipo de ambientes, la gran fluctuación de los niveles de lluvias durante estaciones (Badylak et al,. 2016). En este sentido, durante la época de lluvias, al aumentar las precipitaciones, aumenta el caudal (Itayama et al,. 2015), lo que dificulta que las diatomeas se asienten en el sustrato al ser arrastradas muchas de ellas (Ramírez y Plata-Díaz, 2008).

La mayor riqueza de especies, se observó especialmente en los géneros de Gomphonema, Nitzschia, Fragilaria y Navicula, durante todo el muestreo, que coincide con las riquezas observadas en otros estudios en países tropicales con características similares, como en Colombia (e.g. Vouilloud et al., 2010; Sala et al., 2015), Bolivia (e.g. Morales y Rivera, 2013; Morales et al., 2014), o en el mismo Perú (e.g. Alarcón y Peláez, 2012; Calizaya-Anco et al., 2013, Leiva et al., 2015)

En relación a la abundancia de especies, se observó que Gomphonema rhombicum, Achnanthes minutisima, Gomphonema pumilum var. rigidum, Nitzschia palea, Cocconeis euglypta y Nitzschia media, fueron las más abundantes, representando el $57 \%$ de todos los individuos recolectados e identificados, lo que puede indicar una baja diversidad relacionada con la modificación del ecosistema (Gudmundsdottir et al., 2013; Calapez et al., 2014; Vélez-Azañero et al., 2016), ya que las especies de estos géneros son bastante tolerantes tanto a los cambios en los ecosistemas acuáticos como al aumento de la contaminación (Teittinen et al., 2015), estacionalidad en las precipitaciones (PedrazaGarzón y Donato-Rondón, 2011), cambios en el caudal (Montoya y Ramírez, 2007) y a la desaparición del bosque ribereño (Bixby et al., 2008).
Achnanthes minutissima y Gomphonema rhombicum son las especies más abundantes con respecto al total, siendo la primera la más abundante en la época húmeda, y la segunda la más abundante en la época seca. Esto se relaciona con la gran estacionalidad existente en la cuenca del río Utcubamba, y se puede corroborar con otros estudios que mostraron que Achnanthes minutisima abunda en ambientes con caudales oxigenados (Novais et al., 2015), propios de épocas húmedas con aumentos del caudal y can movimiento del sistema acuático, además de poco contaminados (González, 2012). Por el contrario, Gomphonema rhombicum se desarrolla mucho mejor en ambientes con caudales poco oxigenados propios de épocas secas (Calapez et al., 2014), indicando además cierta tolerancia a la contaminación (Leiva et al., 2015).

\section{CONCLUSIÓN}

En total, fueron colectados 115783 individuos correspondientes a 21 familias, 25 géneros y 112 especies, en los muestreos realizados tanto en la estación seca como en la estación húmeda.

El género más diverso fue Gomphonema, con 18 especies identificadas, además de ser el género más abundante, con un $26 \%$ del total de individuos colectados. Asimismo, la especie Gomphonema rhombicum fue las más abundante, con $17 \%$ de los individuos respecto al total.

La comparativa realizada entre las dos épocas de muestreo, seca en el mes de agosto, y húmeda en el mes de marzo, no muestra una gran similitud tanto a nivel de diversidad como de abundancia, obteniendo la época seca mayores registros para ambas variables debido a que las lluvias en el mes de marzo suponen un factor en el decrecimiento en la comunidad de diatomeas del río Utcubamba.

\section{REFERENCIAS BIBLIOGRÁFICAS}

Alarcón, N. y F. Peláez. “Calidad del agua del río Sendamal (Celendín, Cajamarca, Perú): Determinación mediante uso de diatomeas". Rebiol, 34(2012): 29-37. 
Badylak, S., E. Phlips, N. Dix, J. Hart, A. Srifa, D. Haunert, Z. He, J. Lockwood, P. Stofella, D. Sun y Y. Yang. "Phytoplankton dynamics in a subtropical Tidal creek: Influences of rainfall and water residence time on composition and biomass". Marine and Freshwater Research, 67(2016): 466-482.

Bixby, R.J., J.P. Benstead, M.M. Douglas y C.M. Pringle. "Invasive species impact: Asymmetric interactions between invasive and endemic freshwater snails". Journal of the North American Benthological Society, 27(2008): 509-520.

Blanco, S., L. Ector y E. Bécares. "Epiphytic diatoms as water quality indicators in Spanish shallow lakes". Vie et Milieu, 54(2004): 71-79.

Calapez, A.R., C.L. Elias, S.F.P. Almeida y M.J. Feio. "Extreme drought effects and recovery patterns in the benthic communities of temperate streams". Limnetica, 33(2014): 281-296.

Calizaya-Anco, J., M. Avendaño-Cáceres y I. Delgado-Vargas. "Evaluación de la calidad del agua fluvial con diatomeas (Bacillariophyceae), una experiencia en Tacna, Perú". Revista Peruana de Medicina Experimental y Salud Pública, 30(2013): 58-63.

Céspedes-Vargas, E., G. Umaña-Villalobos y A.M. Silva-Benavides. "Tolerancia de diez especies de diatomeas (Bacillariophyceae) a los factores físico-químicos del agua en el río Sarapiquí, Costa Rica". Revista de Biología Tropical, 64 (2016): 105-115.

CHD (Confederación Hidrográfica del Duero). "Guía de las diatomeas de la cuenca del Duero". Valladolid(España), 2010.

CHE (Confederación Hidrográfica del Ebro). "Metodología para el establecimiento del estado ecológico según la Directiva Marco del Agua en la Confederación Hidrográfica del Ebro". Zaragoza (España), 2005.

Ciutti, F. "Il Monitoraggio dei corsi d'acqua con indicatori algali (Diatomee)". Annali dell'Istituto
Superiore di Sanita, 41(2005): 393-397.

Díaz, C. y N. Maidana. "Diatomeas de los salares Atacama y Punta Negra, II Región - Chile”. Santiago de Chile (Chile), 2005.

González, S.E.H. "Indicadores biológicos de calidad de las aguas superficiales de la subcuenca del río Viejo, utilizando fitobentos (Diatomeas)". Revista Universidady Ciencia, 21(2012): 21-24.

González, S.E.H. "Diatomeas como indicadoras de calidad de agua en la laguna Acahualinca (Managua, Nicaragua)". Revista Agua y Conocimiento, 2(2016): 10-18.

Gudmundsdottir, R., S. Palsson, E.R. Hannesdottir, J.S. Olafsson, G.M. Gislason y B. Moss “Diatoms as indicators: the influences of experimental nitrogen enrichment on diatom assemblages in sub-arctic streams". Ecological Indicators, 32(2013): 74-81.

Itayama, T., P. Hawkins, P. Leelahakriengkrai, S. Kullasoot, N. Whangchai, C. Chitmanat, Y. Peerapornpisal y Z. Kawabata. "Bioassessment of dry season water quality in the Ping river around Chiang Mai City, Thailand". Chiang Mai Journal of Science, 42(2015): 349-366.

Leiva, D., E. Coronel, y F. Corroto. "Uso de diatomeas para medir el impacto de la extracción de agregados en ríos altoandinos, Amazonas, Perú". Revista de Investigación para el Desarrollo Sustentable, 3(2015): 34-42.

Montoya, Y. y J.J. Ramírez. "Variación estructural de la comunidad perifítica colonizadora de sustratos artificiales en la zona de Ritral del río Medellín, Colombia”. Revista de Biología Tropical, 55(2007): 585-593.

Morales, E.A. y S.F. Rivera. "Macrófitas poco frecuentes o desconocidas de la laguna Alalay, Cochabamba, Bolivia”. Acta Nova, 6(2013): 36-52.

Morales, E.A., S.F. Rivera, C.E. Wetzel, M.H. Novais, P.B. Hamilton, L. Hoffmann y L. Ector. "New epiphytic araphid diatoms in the genus 
Ulnaria (Bacillariophyta) from Lake Titicaca, Bolivia”. Diatom research, 29(2014): 41-54.

Novais, M.H., I. Jüttner, B. Van de Vijver, M.M. Morais, L. Hoffmann y L. Ector. "Morphological variability within the Achnanthidium minutissimum species complex (Bacillariophyta): Comparison between the type material of Achnanthes minutissima and related taxa, and new freshwater Achnanthidium species from Portugal”. Phytotaxa, 224 (2015): 101-139.

Ortiz-Castro, M.E. "Caracterización de diatomeas como herramienta para el estudio de la calidad del agua del río Teaone”. Tesis de Ingeniería para optar al grado académico de Ingeniero de Gestión Ambiental, Pontificia Universidad Católica del Ecuador. Esmeraldas (Ecuador), 2015.

Pedraza-Garzón, E y J.C. Donato-Rondón. “Diversity and distribution of diatoms in a mountain stream of the Colombian Andes". Caldasia, 33(2011): 177-191.

Ramírez, A.M. y Y. Plata-Díaz. "Diatomeas perifíticas en diferentes tramos de dos sistemas lóticos de alta montaña (Páramo de Santurbán, Norte De Santander, Colombia) y su relación con las variables ambientales". Acta biol. Colomb, 13(2008): 199-216.

Sala, S.E., A.A. Vouilloud, Y. Plata-Díaz, E. Pedraza y A. Pimienta. "Taxonomía y distribución de diatomeas epilíticas registradas por primera vez en Colombia”. Limnología, 37(2015): 125-141.

Soler, A., M. Pérez, A. Edibelrto y I. Villareal. "Diatomeas del Canal de Panamá: Bioindicadores y otros estudios pioneros”. Panamá (Panamá), 2012.

Teittinen, A., M. Taka, O. Ruth y J. Soininen. "Variation in stream diatom communities in relation to water quality and catchment variables in a boreal, urbanized region". Science of the Total Environment, 530-531(2015): 279-289.
Vélez-Azañero, A., S. Lozano y K. Cáceres-Torres. "Diversidad de fitoplancton como indicador de calidad de agua en la cuenca baja del río Lurín, Lima, Perú". Ecología Aplicada, 15(2016): 69-79.

Vouilloud, A.A., S.E. Sala, M.N. Avellaneda y S.R. Duque. "Diatoms from the Colombian and Peruvian Amazon: The genera Encyonema, Encyonopsis and Gomphonema (Cymbellales: Bacillariophyceae)". Revista de Biología Tropical, 58(2010): 45-62.

Vouilloud, A.A., Y. Plata-Díaz, E. Pedraza, A. Pimienta, S. Heguilor, A. Lamaro y E. Sala. "Distribución de Eunotia Parasiolii (Bacillariophyceae) en ríos neotropicales (Colombia) y su implicancia en la taxonomía de las especies". Hidrobiológica, 26(2016): 241-250.

Yupanqui, C.B.C. y L.A.P. Quispe. "Estudio del fitoplancton en el lago Titicaca." Web Academia ( 2014 ): $1-11$. Recuperado de: http://s3.amazonaws.com/academia.edu.doc uments/36205737/crucero_2014.pdf?AWSA c ces s Key Id=A K I A I W O W Y Y GZ2 Y 53UL3A\&Expires $=1499927634 \&$ Signature $=\mathrm{dMdyN} 5 \mathrm{I}$ 